\title{
Hot Coronae in Local AGN: Present Status and Future Perspectives
}

\author{
Andrea Marinucci ${ }^{1, *}$ (D) , Francesco Tamborra ${ }^{2}$, Stefano Bianchi ${ }^{1}$ (D), Michal Dovčiak ${ }^{2}$ (D), \\ Giorgio Matt ${ }^{1}$ (D), Riccardo Middei ${ }^{1}$ (D) and Alessia Tortosa ${ }^{1,3}$ \\ 1 Dipartimento di Matematica e Fisica, Università degli Studi Roma Tre, via della Vasca Navale 84, \\ 00146 Rome, Italy; bianchi@fis.uniroma3.it (S.B.); matt@fis.uniroma3.it (G.M.); \\ riccardo.middei@uniroma3.it (R.M.); tortosa@fis.uniroma3.it (A.T.) \\ 2 Astronomical Institute of the Czech Academy of Sciences, CZ-14100 Prague, Czech Republic; \\ francesco.tamborra@asu.cas.cz (F.T.) michal.dovciak@asu.cas.cz (M.D.) \\ 3 INAF/Istituto di Astrofisica e Planetologie Spaziali, Via Fosso del Cavaliere, I-00133 Roma, Italy \\ * Correspondence: marinucci@fis.uniroma3.it
}

Received: 2 February 2018; Accepted: 19 March 2018; Published: 4 April 2018

\begin{abstract}
The nuclear X-ray emission in radio-quiet Active Galactic Nuclei (AGN) is commonly believed to be due to inverse Compton scattering of soft UV photons in a hot corona. The radiation is expected to be polarized, the polarization degree depending mainly on the geometry and optical depth of the corona. Nuclear Spectroscopic Telescope Array (NuSTAR) observations are providing for the first time high quality measurements of the coronal physical parameters-temperature and optical depth. We hereby review the NuSTAR results on the coronal physical parameters (temperature and optical depth) and discuss their implications for future X-ray polarimetric studies.
\end{abstract}

Keywords: AGN; X-ray polarimetry; accretion

\section{Introduction}

Active Galactic Nuclei (AGN) represent the perfect astrophysical laboratory to study accretion processes. The great luminosities observed in these objects are due to the efficient conversion of gravitational energy into radiation in an accretion disk surrounding the central supermassive black hole $(\mathrm{SMBH})$. The common paradigm for the geometry of the innermost regions around the central black hole postulates also the presence of a hot corona (the so called two phase model [1,2]). In this scenario, the UV radiation from the accretion disk (emitted as a multi-temperature black body spectrum) is Compton scattered by the hot electrons in the corona, up to X-ray wavelengths. In general, the primary emission of Seyfert galaxies sources can be well approximated as a cutoff power law with a photon index $\Gamma=1.7-2.0$ and a high energy rollover at $\mathrm{E}_{\mathrm{c}}=50-300 \mathrm{keV}$ [3] which strongly depend on the physical properties of the scattering corona, i.e., the optical depth $\tau$ and the electron temperature $\mathrm{kT}_{e}$ [4]. Nonetheless, the reprocessing of the nuclear radiation of the AGN from the circumnuclear environment makes the measurement of the slope of the continuum and of the cutoff energy degenerate with other physical parameters, like the amount of radiation reflected by circumnuclear matter, which produces a Compton hump at $\sim 30 \mathrm{keV} \mathrm{[5,6].} \mathrm{A} \mathrm{broad} \mathrm{spectral} \mathrm{coverage,} \mathrm{from} 0.5 \mathrm{up}$ to $100 \mathrm{keV}$ is therefore fundamental to discriminate between the different components of an X-ray spectrum, and to measure the parameters of the intrinsic nuclear emission. In this context, the Nuclear Spectroscopic Telescope Array (NuSTAR). has played a very important role, with its operating band up to $79 \mathrm{keV}$ and an unprecedented sensitivity above $10 \mathrm{keV}$.

In the past, several works have shown that in the hot corona, assumed to be a slab above the disk, the high-energy cutoff is $2-3$ times the electron temperature $[7,8]$. However, in the past few 
years, many theoretical efforts have been spent on proposing different geometries (patchy coronae, outflowing coronae $[9,10])$ and on investigating the general relativistic effects occurring in a lamp-post geometry (in which the X-ray source is assumed to be very compact and along the black hole axis [11,12]). Despite the growing number of models and codes for Comptonization available to the scientific community, no significant discrimination between geometrical models has been possible so far based solely on spectroscopy, even in the brightest, unobscured sources with very accurate high energy cutoff measurements: IC 4329A ( $E_{\mathrm{c}}=186 \pm 14 \mathrm{keV}$ [13]), MCG-5-23-16 $\left(\mathrm{E}_{\mathrm{c}}=116_{-5}^{+6} \mathrm{keV}\right.$ [14]), SWIFT J2127.4+5654 $\left(\mathrm{E}_{\mathrm{c}}=108_{-10}^{+11} \mathrm{keV}\right.$ [15]), 3C $382\left(\mathrm{E}_{\mathrm{c}}=214_{-63}^{+147} \mathrm{keV}\right.$ [16]). Thanks to the recently approved IXPE satellite (expected to be launched in early $2021[17,18]$ ), X-ray polarimetry will be the new pair of eyes that will allow us to prefer one coronal geometry to another, at least in the brightest, unobscured AGN.

In the following, we will review the present status of the hot coronal measurements in local AGN, discuss some applications of a new model for Comptonization in astrophysics (MoCA [19]), and present future perspectives in the framework of X-ray polarimetry.

\section{Recent Results}

The high energy spectral curvature of AGN has been largely investigated since the launch of X-ray satellites operating above $10 \mathrm{keV}$, despite large degeneracy between the slope of the primary continuum, the cutoff energy and the amount of Compton scattered radiation by the circumnuclear material, mainly due to the fact that BeppoSAX, Suzaku, INTEGRAL and Swift cannot focus hard X-ray photons and they are therefore strongly background-dominated. BeppoSAX was the first satellite which had been used for this kind of studies $[8,20]$ showing a relation between the photon index of the primary power law and the high energy curvature [21], likely due to the limited sensitivity of the instrument at high energies. With the advent of INTEGRAL many other works described the high energy behavior of type 1 and type 2 AGN, in particular when XMM-Newton, Suzaku or Chandra were used to cover the energy band below $10 \mathrm{keV}$ [22-25]. Some of these works showed that there is a degeneracy between the cutoff energy and the Compton reflection fraction (commonly indicated as $R$ ) [25] while average values of $\Gamma=1.76_{-0.24}^{+0.22}, \mathrm{E}_{\mathrm{C}}=106_{-61}^{+186} \mathrm{keV}, R=1.5_{-1.0}^{+1.5}$ are retrieved when only Type 1 AGN are taken into account [22]. A recent work with Swift-BAT on 838 AGN confirmed these results, with cutoff energies in the range $50-300 \mathrm{keV}$ for the whole non-blazar AGN sample [26].

NuSTAR [27], launched in June 2012, is the first telescope focusing X-rays above $10 \mathrm{keV}$ up to $79 \mathrm{keV}$. It has led to a number of results about the high energy emission of AGN. Recent papers [3,28] have collected and discussed the high energy cutoff measurements in the first years of NuSTAR, for about twenty unobscured nearby Seyfert galaxies (with a column density along the line of sight $\mathrm{N}_{\mathrm{H}}<6 \times 10^{22} \mathrm{~cm}^{-2}$ ). The model used to fit the X-ray spectra of the sample is generally composed of a primary continuum (a cutoff power law) and Compton reflection from distant material and from the accretion disk. The reprocessed emission is generally modeled using XILLVER and RELXILL components $[29,30]$ : while the former can be used to retrieve information about the Iron abundance, inclination angle and ionization state of the reflecting matter far from the nucleus, the latter includes relativistic effects due to reflection coming from the innermost regions of the accretion disk, in proximity of the central black hole. The range of photon indices and cutoffs previously found with BeppoSAX, INTEGRAL and Swift-BAT has been confirmed $\left(\Gamma=1.7-2.0, \mathrm{E}_{\mathrm{c}}=50-300 \mathrm{keV}\right)$ but the degeneracy between the two observables has been finally broken (Figure 2 in [3]). The lowest values for the high energy turnover are the ones found in Ark $564\left(\mathrm{E}_{\mathrm{c}}=42 \pm 3 \mathrm{keV}\right.$ [31]) and GRS 1734-292 $\left(\mathrm{E}_{\mathrm{c}}=53 \pm 10 \mathrm{keV}\right.$ [32]), and the highest in NGC $5506\left(\mathrm{E}_{\mathrm{c}}=720_{-190}^{+130} \mathrm{keV}\right.$ [33]). When more physical Comptonization models such as COMPTT, COMPPS, NTHCOMP [34-37] are taken into account (and the primary cutoff power law hence removed) values for the optical depth and electron temperature of the hot corona can be inferred with very good statistical accuracy. COMPTT is an analytic model that describes the Comptonization spectrum of seed optical/UV photons scattering off purely thermal electrons in the corona, while in COMPPS exact numerical solutions of the radiative transfer equation 
are taken into account, as well as Compton reflection from neutral material. At last, NTHCOMP allows to retrieve a photon index $\Gamma$ of the Comptonized spectrum for a given pair of temperatures for the corona $\left(\mathrm{kT}_{e}\right)$ and for the accretion disk $\left(\mathrm{kT}_{b b}\right)$. These models assume a geometry in which the corona is extended above the accretion disk and distributed as a slab or a sphere (Figure 1, panels (a) and (b), respectively). In a slab configuration the range of optical depth values is $\tau=0.01-2.5$, with a $\mathrm{kT}_{e}=10-300 \mathrm{keV}$ and these parameters do not correlate with physical quantities of the system such as the black hole mass, the luminosity or the accretion rate [3], as previously discussed in the past using INTEGRAL [22].

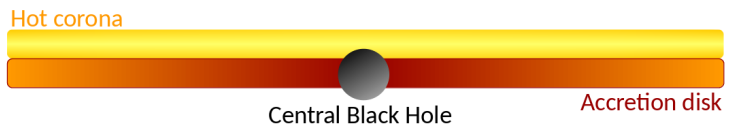

(a)

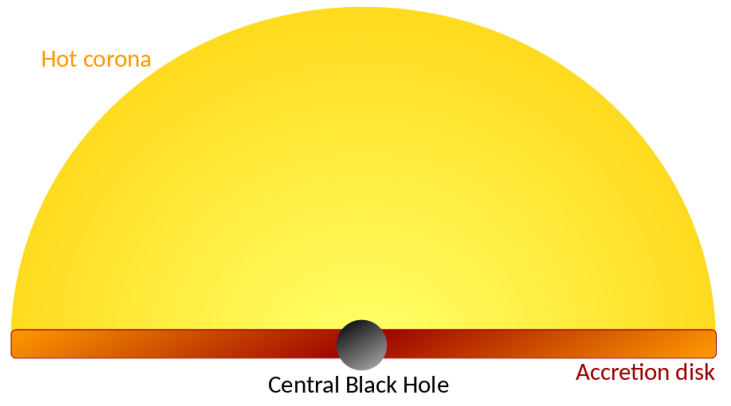

(b)

Figure 1. (a) Slab geometry for a hot corona distributed above an accretion disk surrounding the central black hole; (b) Spherical geometry for a hot corona.

Taking into account both slab and spherical geometries the only statistically significant correlation is between the optical depth and the electron temperature, following the relations presented in [3]:

$$
\begin{gathered}
\text { SLAB }: \log \left(\mathrm{kT}_{e}\right)=(-0.7 \pm 0.1) \log (\tau)+(1.60 \pm 0.06) \\
\text { SPHERE }: \log \left(\mathrm{kT}_{e}\right)=(-0.7 \pm 0.2) \log (\tau)+(1.8 \pm 0.1)
\end{gathered}
$$

A fixed disk-corona geometry in radiative balance cannot be invoked to explain the observed $\tau-\mathrm{kT}_{e}$ anti-correlation in a classical two-phase scenario, in which the bulk of energy is dissipated in the corona and the disk is mostly passive [1,2]. Different geometries for the accretion flow and a variation of the coronal heating/cooling ratio are therefore needed to explain the $\tau-\mathrm{kT}_{e}$ distribution in the whole sample [3,38]. Different works on the same sources have also shown that under the assumption that the corona is compact, only extended for a few gravitational radii, two interesting parameters such as the compactness parameter $(l \propto L / R[39,40])$ and the adimensional electron temperature $\left(\Theta=\mathrm{kT}_{e} / m_{e} c^{2}\right)$ can be introduced. The majority of the objects lie very close to the forbidden region of electron-positron pair production in the $\Theta-l$ diagram, indicating that pair production and annihilation act as a sort of thermostat for AGN coronae [41]. The boundary curve depends from the geometry of the plasma but also from its composition. In presence of non-thermal particles the hybrid, Compton scattering plasma shows a wider range of allowed temperatures [42].

\section{From X-Ray Spectroscopy to Polarimetry}

In none of the objects observed with NuSTAR, simultaneously to XMM, Chandra, Suzaku, Swift, and included in the catalogs mentioned above [3,28,41,42], X-ray spectroscopy has led to a clear discrimination between different coronal geometries. On the other hand, Compton scattering, as any scattering mechanism, will produce a polarization signal which is strongly sensitive to the geometry of the scattering material. The IXPE and eXTP satellites $[17,18,43]$ will be therefore crucial to the study of the coronae in the brightest, unobscured AGN. The Imaging X-ray Polarimetry Explorer (IXPE) has been selected by NASA as a SMall EXplorer Mission (SMEX) for a launch in early 2021. With a polarization sensitivity of 1.8\% MDP (Minimum Detectable Polarization) in $300 \mathrm{ks}$, for a source 


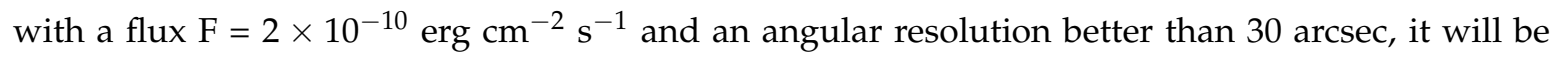
the first X-ray imaging polarimeter on orbit. On the other hand, the enhanced X-ray Timing and Polarimetry mission (eXTP) has been proposed to the Chinese Academy of Sciences and selected in 2011 as one of 8 background missions. Its operating energy band is $2-10 \mathrm{keV}$ and its sensitivity will be a factor 5 better in observing time than IXPE, with a comparable angular resolution. In this context, a Comptonization code which includes both general and special relativity effects as well as polarization has been recently released (MoCA, a Monte Carlo code for Comptonization in Astrophysics [19,44]). Compared to different codes in the literature, the energy-dependent Klein-Nishina cross section is taken into account [45] and different, multiple geometries are implemented [46]. We show in Figure 2 (left panel) the 2-400 keV spectrum simulated for a slab corona with $\mathrm{kT}_{e}=100 \mathrm{keV}, \tau=1.0$, covering the whole disk from 6 to 500 gravitational radii, with general relativistic effects switched off, for computational time purposes. The black hole mass and the accretion rate of the source are $\mathrm{M}_{\mathrm{bh}}=1.5 \times 10^{8} \mathrm{M}_{\text {sun }}$ and $10 \%$ of the Eddington luminosity, respectively. The data are then fitted in red with a cutoff power law and clear residuals are present above $\sim 50 \mathrm{keV}$, indicating a large deviation from MoCA simulations, highlighting the importance of using proper Comptonization models instead of a purely phenomenological cutoff power law.

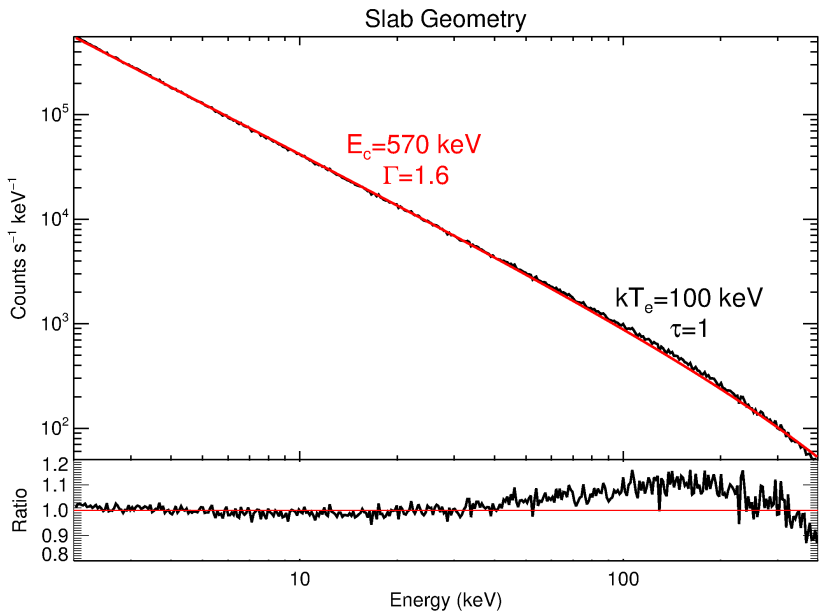

(a)

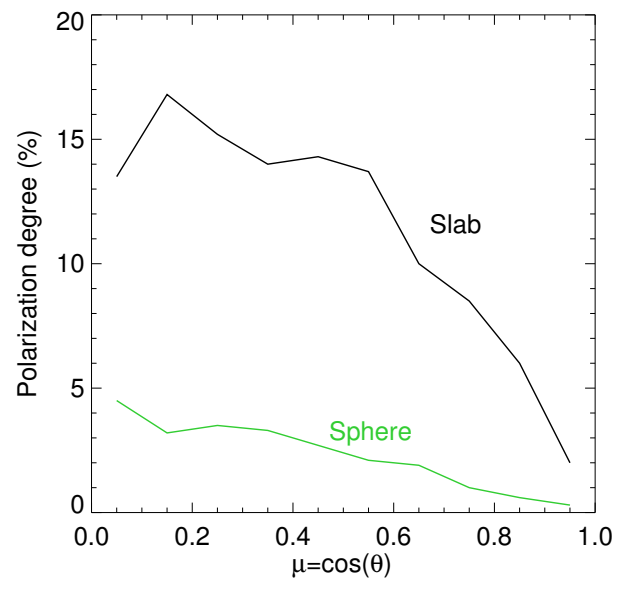

(b)

Figure 2. (a) The $\mathrm{X}$-ray spectrum simulated for a slab corona with $\mathrm{kT}_{e}=100 \mathrm{keV}, \tau=1.0$ with Monte Carlo code for Comptonization in Astrophysics (MoCA) (in black), fitted with a power law model (in red) is shown. Residuals are reported in the bottom panel; (b) The polarization degree is plotted against the cosine of the inclination angle for two different geometries (see text for details).

One of the brightest objects in the NuSTAR sample of unobscured sources is IC 4329A, with a $2-10 \mathrm{keV}$ flux $\mathrm{F} \simeq 1 \times 10^{-10} \mathrm{erg} \mathrm{cm}^{-2} \mathrm{~s}^{-1}$ [3,41,42]. A detailed fit to the simultaneous Suzaku-NuSTAR spectra with COMPTT showed that a slab coronal geometry (with parameters $\left.\mathrm{kT}_{e}=61 \pm 1 \mathrm{keV}, \tau=0.68 \pm 0.02\right)$ and a spherical one $\left(\mathrm{kT}_{e}=50_{-3}^{+6} \mathrm{keV}, \tau=2.34_{-0.21}^{+0.16}\right)$ are statistically equivalent to reproduce the data set [13]. In Figure 2 (b panel) we show the expected polarization degree for the two physical scenarios simulated with MoCA, using $5 \times 10^{8}$ input photons initially unpolarized. The polarization degree, integrated between 2 and $8 \mathrm{keV}$ (i.e., the IXPE operating energy band), is plotted against the cosine of the inclination angle. The polarization is always vertical, i.e., with the polarization vector parallel to the projection on the plan of the sky of the disk axis. When the system is observed face-on $(\mu=1)$ it is perfectly symmetric and the polarization degree approaches zero. When it is edge-on the polarization degree increases up to $15 \%$ for the slab and $5 \%$ for the sphere, being the latter a much more symmetrical geometry than the slab and therefore producing a much weaker polarization signal. These simulations show that for a source like IC 4329A, with an inclination angle of 60 degrees for its accretion disk [13], the coronal geometry can be clearly inferred. For this 
source, a Minimum Detectable Polarization (MDP) of $2 \%$ with IXPE can be obtained with an exposure time of approximately $450 \mathrm{ks}$.

\section{Conclusions}

We have reviewed the current status of the coronal parameters measurements in local AGN, after the launch of $N u S T A R$, highlighting commonalities and differences with previous analyses performed with background-dominated satellites. Unfortunately, X-ray spectroscopy has led to a clear discrimination between different coronal geometries in none of the objects observed. Thanks to the IXPE and eXTP satellites (expected to be launched in early 2021 and 2025), X-ray polarimetry will be the new pair of eyes that will allow us to prefer one coronal geometry to another. Taking advantage of the MoCA Comptonization code, we performed simulations in two coronal geometries, retrieving different polarization degrees (up to 15\% for the slab and 5\% for the sphere). In the very near future, we will therefore be able to distinguish between different coronal geometries responsible for the X-ray nuclear continuum, at least in the brightest, unobscured AGN.

Acknowledgments: We acknowledge financial support the European Union Seventh Framework Programme (FP7/2007-2013) under grant agreement n.312789. A.M., G.M. and A.T. acknowledge financial support from the Italian Space Agency under grant ASI/INAF I/037/12/0-011/13. A.M., S.B., G.M., R.M., A.T. acknowledge financial support from Italian Space Agency (ASI) under grants ASI/INAF 2015-034-R.0 and 2017-12-H.0.

Author Contributions: A.M. performed numerical simulations with MoCA and wrote the paper. F.T. developed the MoCA Comptonization code and R.M, A.T. directly contributed and worked on some of the catalogs discussed in the article. S.B., M.D., G.M. contributed to many works included in the paper and provided suggestions to improve the general shape of the manuscript.

Conflicts of Interest: The authors declare no conflicts of interest.

\section{References and Notes}

1. Haardt, F.; Maraschi, L. A two-phase model for the X-ray emission from Seyfert galaxies. Astrophys. J. 1991, 380, L51-L54.

2. Haardt, F.; Maraschi, L.; Ghisellini, G. A model for the X-ray and ultraviolet emission from Seyfert galaxies and galactic black holes. Astrophys. J. 1994, 432, L95-L99.

3. Tortosa, A.; Bianchi, S.; Marinucci, A.; Matt, G.; Petrucci, P.O. A NuSTAR census of coronal parameters in Seyfert galaxies. arXiv 2018, arXiv:1801.04456.

4. Rybicki, G.B.; Lightman, A.P. Radiative Processes in Astrophysics; Wiley-Interscience: New York, NY, USA, 1979.

5. Matt, G.; Perola, G.C.; Piro, L. The iron line and high energy bump as X-ray signatures of cold matter in Seyfert 1 galaxies. Astron. Astrophys. 1991, 247, 25-34.

6. George, I.M.; Fabian, A.C. X-ray reflection from cold matter in active galactic nuclei and X-ray binaries. Mon. Not. R. Astron. Soc. 1991, 249, 352-367.

7. Petrucci, P.O.; Haardt, F.; Maraschi, L.; Grandi, P.; Matt, G.; Nicastro, F.; Piro, L.; Perola, G.C.; De Rosa, A. Testing Comptonizing Coronae on a Long BeppoSAX Observation of the Seyfert 1 Galaxy NGC 5548. Astrophys. J. 2000, 540, 131.

8. Petrucci, P.O.; Haardt, F.; Maraschi, L.; Grandi, P.; Malzac, J.; Matt, G.; Nicastro, F.; Piro, L.; Perola, G.C.; De Rosa, A. Testing Comptonization Models Using BeppoSAX Observations of Seyfert 1 Galaxies. Astrophys. J. 2001, 556, 716.

9. Wilkins, D.R.; Gallo, L.C. The Comptonization of accretion disc X-ray emission: Consequences for X-ray reflection and the geometry of AGN coronae. Mon. Not. R. Astron. Soc. 2015, 448, 703-712.

10. Wilkins, D.R.; Gallo, L.C.; Grupe, D.; Bonson, K.; Komossa, S.; Fabian, A.C. Flaring from the supermassive black hole in Mrk 335 studied with Swift and NuSTAR. Mon. Not. R. Astron. Soc. 2015, 454, 4440.

11. Tamborra, F.; Papadakis, I.; Dovčiak, M.; Svoboda, J. On the high energy cut-off of accreting sources: Is general relativity relevant? Mon. Not. R. Astron. Soc. 2018, 475, 2045-2050.

12. Dovčiak, M.; Done, C. Minimum X-ray source size for a lamppost corona in light-bending models for AGN. arXiv 2015, arXiv:1507.05564. 
13. Brenneman, L.W.; Madejski, G.; Fuerst, F.; Matt, G.; Elvis, M.; Harrison, F.A.; Ballantyne, D.R.; Boggs, S.E.; Christensen, F.E.; Craig, W.W.; et al. The Broad-band X-Ray Spectrum of IC 4329A from a Joint NuSTAR/Suzaku Observation. Astrophys. J. 2014, 788, 90-95.

14. Baloković, M.; Matt, G.; Harrison, F.A.; Zoghbi, A.; Ballantyne, D.R.; Boggs, S.E.; Christensen, F.E.; Craig, W.W.; Esmerian, C.J.; Fabian, A.C.; et al. Coronal Properties of the Seyfert 1.9 Galaxy MCG-05-23-016 Determined from Hard X-Ray Spectroscopy with NuSTAR. Astrophys. J. 2015, 800, 62.

15. Marinucci, A.; Matt, G.; Kara, E.; Miniutti, G.; Elvis, M.; Arevalo, P.; Ballantyne, D.R.; Balokovic, M.; Bauer, F.; Brenneman, L.; et al. Simultaneous NuSTAR and XMM-Newton 0.5-80 keV spectroscopy of the narrow-line Seyfert 1 galaxy SWIFT J2127.4+5654. Mon. Not. R. Astron. Soc. 2014, 440, 2347.

16. Ballantyne, D.R.; Bollenbacher, J.M.; Brenneman, L.W.; Madsen, K.K.; Balokovic, M.; Boggs, S.E.; Christensen, F.E.; Craig, W.W.; Gandhi, P.; Hailey, C.J.; et al. NuSTAR Reveals the Comptonizing Corona of the Broad-line Radio Galaxy 3C 382. Astrophys. J. 2014, 794, 210.

17. Weisskopf, M.C.; Ramsey, B.; O’Dell, S.; Tennant, A.; Elsner, R.; Soffita, P.; Bellazzini, R.; Costa, E.; Kolodziejczak, J.; Kaspi, V.; et al. The Imaging X-ray Polarimetry Explorer (IXPE). Proc. SPIE 2016, 9905, 990517.

18. Weisskopf, M.C.; Ramsey, B.; O’Dell, S.; Tennant, A.; Elsner, R.; Soffita, P.; Bellazzini, R.; Costa, E.; Kolodziejczak, J.; Kaspi, V.; et al. The Imaging X-ray Polarimetry Explorer (IXPE). Results Phys. 2016, 6, 1179-1180.

19. Tamborra, F.; et al. In preparation.

20. Dadina, M. BeppoSAX observations in the $2-100 \mathrm{keV}$ band of the nearby Seyfert galaxies: An atlas of spectra. Astron. Astrophys. 2007, 461, 1209-1252.

21. Perola, G.C.; Matt, G.; Cappi, M.; Fiore, F.; Guainazzi, M.; Maraschi, L.; Petrucci, P.O.; Piro, L. Compton reflection and iron fluorescence in BeppoSAX observations of Seyfert type 1 galaxies. Astron. Astrophys. 2002, 389, 802-811.

22. Molina, M.; Bassani, L.; Malizia, A.; Stephen, J.B.; Bird, A.J.; Dean, A.J.; Panessa, F.; De Rosa, A.; Landi, R. The INTEGRAL complete sample of type 1 AGN. Mon. Not. R. Astron. Soc. 2009, 399, 1293-1306.

23. Panessa, F.; de Rosa, A.; Bassani, L.; Bazzano, A.; Bird, A.; Landi, R.; Malizia, A.; Miniutti, G.; Molina, M.; Ubertini, P. Narrow-line Seyfert 1 galaxies at hard X-rays. Mon. Not. R. Astron. Soc. 2011, 417, 2426.

24. De Rosa, A.; Panessa, F.; Bassani, L.; Bazzano, A.; Bird, A.; Landi, R.; Malizia, A.; Molina, M.; Ubertini, P. Broad-band study of hard X-ray-selected absorbed active galactic nuclei. Mon. Not. R. Astron. Soc. 2012, 420, 2087-2101.

25. Molina, M.; Bassani, L.; Malizia, A.; Stephen, J.B.; Bird, A.J.; Bazzano, A.; Ubertini, P. Hard-X-ray spectra of active galactic nuclei in the INTEGRAL complete sample. Mon. Not. R. Astron. Soc. 2013, 433, 1687-1700.

26. Ricci, C.; Trakhtenbrot, B.; Koss, M.J.; Ueda, Y.; Delvecchio, I.; Treister, E.; Schawinski, K.; Paltani, S.; Oh, K.; Lamperti, I.; et al. BAT AGN Spectroscopic Survey. V. X-Ray Properties of the Swift/BAT 70-month AGN Catalog. Astrophys. J. 2017, 233, 17.

27. Harrison, F.A.; Craig, W.W.; Christensen, F.E.; Hailey, C.J.; Zhang, W.W.; Boggs, S.E.; Stern, D.; Cook, W.R.; Forster, K.; Giommi, P.; et al. The Nuclear Spectroscopic Telescope Array (NuSTAR) High-energy X-Ray Mission. Astrophys. J. 2013, 770, 103.

28. Marinucci, A.; Tortosa, A. The coronal parameters of local Seyfert galaxies. Astron. Nachrichten 2016, 337 , 490-494.

29. Garcia, J.; Dauser, T.; Lohfink, A.; Kallman, T.R.; Steiner, J.F.; McClintock, J.E.; Brenneman, L.; Wilms, J.; Eikmann, W.; Reynolds, C.S.; et al. Improved Reflection Models of Black Hole Accretion Disks: Treating the Angular Distribution of X-Rays. Astrophys. J. 2014, 782, 76.

30. Dauser, T.; Garcia, J.; Parker, M.L.; Fabian, A.C.; Wilms, J. The role of the reflection fraction in constraining black hole spin. Mon. Not. R. Astron. Soc. 2014, 444, L100-L104.

31. Kara, E.; Garcia, J.A.; Lohfink, A.; Fabian, A.C.; Reynolds, C.S.; Tombesi, F.; Wilkins, D.R. The high-Eddington NLS1 Ark 564 has the coolest corona. Mon. Not. R. Astron. Soc. 2017, 468, 3489.

32. Tortosa, A.; Marinucci, A.; Matt, G.; Bianchi, S.; La Franca, F.; Ballantyne, D.R.; Boorman, P.G.; Fabian, A.C.; Farrah, D.; Fuerst, F.; et al. Broad-band X-ray spectral analysis of the Seyfert 1 galaxy GRS 1734-292. Mon. Not. R. Astron. Soc. 2017, 466, 4193. 
33. Matt, G.; Balokovic, M.; Marinucci, A.; Ballantyne, D.R.; Boggs, S.E.; Christensen, F.E.; Comastri, A.; Craig, W.W.; Gandhi, P.; Hailey, C.J.; et al. The hard X-ray spectrum of NGC 5506 as seen by NuSTAR. Mon. Not. R. Astron. Soc. 2015, 447, 3029-3033.

34. Titarchuk, L. Generalized Comptonization models and application to the recent high-energy observations. Astrophys. J. 1994, 434, 570-586.

35. Poutanen, J.; Svensson, R. The Two-Phase Pair Corona Model for Active Galactic Nuclei and X-Ray Binaries: How to Obtain Exact Solutions. Astrophys. J. 1996, 470, 249.

36. Zdziarski, A.A.; Johnson, W.N.; Magdziarz, P. Broad-band ?-ray and X-ray spectra of NGC 4151 and their implications for physical processes and geometry. Mon. Not. R. Astron. Soc. 1996, 283, 193.

37. Zycki, P.T.; Done, C.; Smith, D.A. The 1989 May outburst of the soft X-ray transient GS 2023+338 (V404 Cyg). Mon. Not. R. Astron. Soc. 1999, 309, 561-575.

38. Petrucci, P.O.; Ursini, F.; De Rosa, A.; Bianchi, S.; Cappi, M.; Matt, G.; Dadina, M.; Malzac, J. Testing warm Comptonization models for the origin of the soft X-ray excess in AGN. Astron. Astrophys. 2018, 611, A59.

39. Cavaliere, A.; Morrison, P. Extreme nonthermal radiation from active galactic nuclei. Astrophys. J. 1980, 238, L63-L66.

40. Guilbert, P.; Fabian, A.; Rees, M. Spectral and variability constraints on compact sources. Mon. Not. R. Astron. Soc. 1983, 205, 593-603.

41. Fabian, A.C.; Lohfink, A.; Kara, E.; Parker, M.L.; Vasudevan, R.; Reynolds, C.S. Properties of AGN coronae in the NuSTAR era. Mon. Not. R. Astron. Soc. 2015, 451, 4375-4383.

42. Fabian, A.C.; Lohfink, A.; Belmont, R.; Malzac, J.; Coppi, P. Properties of AGN coronae in the NuSTAR era- II. Hybrid plasma. Mon. Not. R. Astron. Soc. 2017, 467, 2566-2570.

43. Zhang, S.N.; Feroci, M.; Santangelo, A.; Dong, Y.W.; Feng, H.; Lu, F.J.; Nandra, K.; Wang, Z.S.; Zhang, S.; Bozzo, E.; et al. eXTP_Enhanced X-ray Timing and Polarimetry Mission. Proc. SPIE 2016, 9905, 20170006127.

44. Middei, R.; et al. In preparation.

45. Schnittman, J.D.; Krolik, J.H. X-ray Polarization from Accreting Black Holes: Coronal Emission. Astrophys. J. 2010, 712, 908-924.

46. Beheshtipour, B.; Krawczynski, H.; Malzac, J. The X-Ray Polarization of the Accretion Disk Coronae of Active Galactic Nuclei. Astrophys. J. 2017, 850, 14.

(C) 2018 by the authors. Licensee MDPI, Basel, Switzerland. This article is an open access article distributed under the terms and conditions of the Creative Commons Attribution (CC BY) license (http:/ / creativecommons.org/licenses/by/4.0/). 